\title{
Conformal Quasicrystals and Holography
}

\author{
Latham Boyle $\odot,{ }^{1}$ Madeline Dickens, ${ }^{2}$ and Felix Flicker ${ }^{2,3}$ \\ ${ }^{1}$ Perimeter Institute for Theoretical Physics, Waterloo, Ontario N2L 2Y5, Canada \\ ${ }^{2}$ Department of Physics, University of California, Berkeley, California 94720, USA \\ ${ }^{3}$ Rudolf Peierls Centre for Theoretical Physics, University of Oxford, Department of Physics, \\ Clarendon Laboratory, Parks Road, Oxford, OX1 3PU, United Kingdom
}

(Received 18 May 2018; revised manuscript received 22 August 2019; published 14 January 2020)

\begin{abstract}
Recent studies of holographic tensor network models defined on regular tessellations of hyperbolic space have not yet addressed the underlying discrete geometry of the boundary. We show that the boundary degrees of freedom naturally live on a novel structure, a "conformal quasicrystal," that provides a discrete model of conformal geometry. We introduce and construct a class of one-dimensional conformal quasicrystals and discuss a higher-dimensional example (related to the Penrose tiling). Our construction permits discretizations of conformal field theories that preserve an infinite discrete subgroup of the global conformal group at the cost of lattice periodicity.
\end{abstract}

DOI: 10.1103/PhysRevX.10.011009

Subject Areas: Condensed Matter Physics,
Interdisciplinary Physics,
String Theory

\section{INTRODUCTION}

A central topic in theoretical physics over the past two decades has been holography: the idea that a quantum theory in a bulk space may be precisely dual to another living on the boundary of that space. The most concrete and widely studied realization of this idea has been the AdS/ CFT correspondence [1-3], in which a gravitational theory living in a $(d+1)$-dimensional negatively curved bulk spacetime is dual to a nongravitational theory living on its $d$-dimensional boundary. Over the past decade, investigations of this duality have yielded mounting evidence that spacetime and its geometry can in some sense be regarded as emergent phenomena, reflecting the entanglement pattern of some other underlying quantum degrees of freedom (d.o.f.) [4].

Recently, physicists have been interested in the possibility that discrete models of holography would permit them to understand this idea in greater detail, by bringing in the tools of condensed matter physics and quantum information theory-much as lattice gauge theory led to conceptual and practical progress in understanding gauge theories in the continuum [5]. Recent years have seen a surge of interest in discrete models of holography based on tensor networks (TNs). Such holographic TNs relate the quantum d.o.f. living on a discretized $(d+1)$-dimensional hyperbolic

Published by the American Physical Society under the terms of the Creative Commons Attribution 4.0 International license. Further distribution of this work must maintain attribution to the author(s) and the published article's title, journal citation, and DOI. space to corresponding d.o.f. on the discretized $d$-dimensional boundary of that space. They bridge condensed matter physics, quantum gravity, and quantum information.

In condensed matter physics, holographic TNs provide a computationally efficient description of the highly entangled ground states of quantum many-body systems (particularly scale-invariant systems or systems near their critical points). The entanglement pattern is described by tensors living on an emergent discrete hyperbolic geometry in one dimension higher [6-11]. On the quantum gravity side, the TNs are a kind of UV regulator of the physics in the bulk, and a proposed way to represent the fact that spacetime and its geometry may be regarded as emergent phenomena, reflecting the entanglement pattern of some other underlying quantum d.o.f. [12-24]. Meanwhile, quantum information theory provides a unifying language for these studies in terms of entanglement, quantum circuits, and quantum error correction [25].

These investigations have gradually clarified our understanding of the discrete geometry in the bulk. There has been a common expectation, based on an analogy with AdS/CFT [1-3], that TNs living on discretizations of a hyperbolic space define a lattice state of a critical system on the boundary and vice versa. Initially, this led Swingle to interpret Vidal's Multiscale Entanglement Renormalization Ansatz (MERA) as a discretized version of a time slice of the AdS geometry [7,12]. However, MERA has a preferred causal direction, while any discretization of a spacelike manifold should not. To fix this issue, it was proposed that MERA was related first to a different object known as the kinematic space of $\operatorname{AdS}[18,26]$ and, more recently, to a lightlike geometry [27]. 
Meanwhile, new TN models (e.g., the holographic quantum error-correcting codes of Ref. [16], the hyperinvariant networks of Ref. [21], or the matchgate networks of Ref. [24]) were introduced to more adequately capture AdS. The key feature of these new TNs is that they live on regular tilings of hyperbolic space [28]. The symmetries of such a tessellation form an infinite discrete subgroup of the continuous symmetry group of the AdS time slice, much as the symmetries of an ordinary 3D lattice or crystal form an infinite discrete subgroup of the continuous symmetry group of 3D Euclidean space. Physically, such TNs represent a discretization of the continuous bulk space, much as a crystal represents a discretization of a continuum material. In this paper, we restrict our attention to TNs of this type.

Despite progress in describing the discrete bulk geometry, the question of which discrete geometric spaces are suitable for the boundary has received little attention. In a discrete model of the AdS/CFT correspondence, we expect to be able to construct the discrete boundary geometry entirely from the data of the bulk tessellation, in analogy with the continuum case, where it is well known that the data of any asymptotically AdS spacetime define a conformal manifold on its boundary [2]. While this expectation seems natural, we are unaware of a corresponding discrete construction in the literature. In condensed matter physics, the discrete boundary geometry is typically assumed to be a periodic lattice; but, as we observe here, there is no natural way in which an ordinary regular tessellation of hyperbolic space defines a periodic lattice on its boundary, and no natural way for the discrete symmetries of the bulk to act on such a boundary lattice $[29,31]$. Thus, we expect that, in implementing a discrete version of AdS/CFT, it will be natural to replace the periodic boundary lattice with a different discrete object. Such a replacement is reasonable, from a Wilsonian viewpoint, as the choice of underlying lattice becomes irrelevant at a critical point [32].

In this paper, we argue that the d.o.f. on the boundary of a regular tessellation of hyperbolic space naturally live on a remarkable structure-a "conformal quasicrystal" (CQC) - built entirely from the data of the bulk tessellation. This provides a new clue about the type of boundary theory that should appear in a discrete version of holography. Far from being a simple periodic lattice, each CQC locally resembles a self-similar quasicrystal [33-37] (like the Penrose tiling [38], shown in Fig. 1) and possesses discrete symmetries worthy of a discretization of a conformal manifold. In this paper, we focus on the $d=1$ case but with a view towards higher dimensions (which we discuss briefly near the end). We propose to use our framework to construct discretizations of CFTs that preserve an infinite discrete subgroup of the global conformal group at the cost of exact discrete translation invariance (which is replaced by quasitranslational invariance). We end with suggestions for future research and a discussion of how our results will hopefully

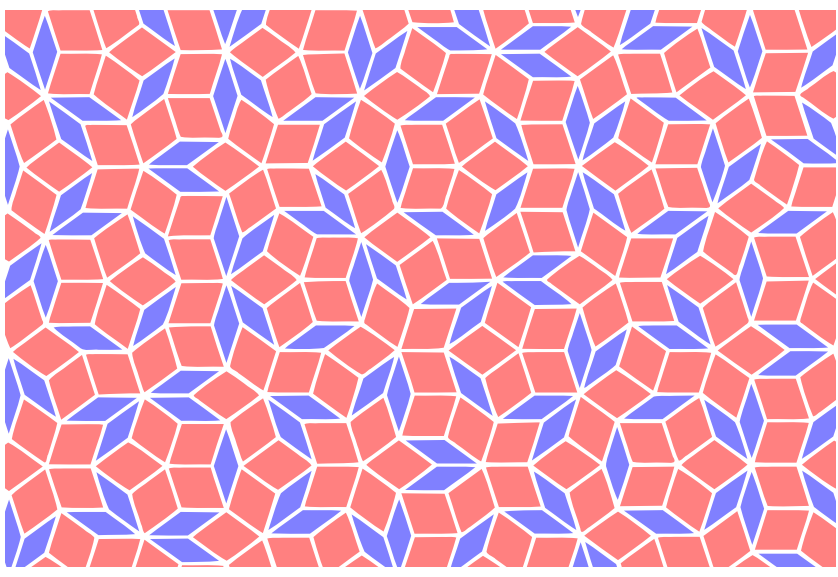

FIG. 1. The Penrose tiling [35]. The two different tiles (red and blue) cover the entire Euclidean plane in an aperiodic manner without gaps. Centers of approximate fivefold symmetry can be seen. The tiling can be created through a cut-and-project method from a five-dimensional hypercubic lattice or a four-dimensional $A_{4}$ lattice, or by applying "inflation rules" to a finite-size starting configuration [33-35].

provide an important clue in the ongoing effort to formulate a discrete version of holography, and also lead to an improved analytical and numerical understanding of the structure of condensed matter systems at their critical points, and other conformally invariant systems.

\section{QUASICRYSTALS IN $\boldsymbol{d}=\mathbf{1}$}

We begin by briefly introducing self-similar quasicrystals (SSQCs) in $d=1$ (see Refs. [33-37] for further details). For general $d$, a SSQC may be thought of as a special quasiperiodically ordered set of discrete points in Euclidean space (like the vertices in an infinite 2D Penrose tiling, shown in Fig. 1). The same SSQC can be constructed in two very different ways: either (i) by a cut-and-project method based on taking an irrationally sloped slice through a periodic lattice in a higher-dimensional Euclidean space and projecting nearby points onto the slice, or (ii) by recursive iteration of an appropriate substitution (or "inflation") rule. Restricting to $d=1$ in the following sections, we exploit the second (inflation) perspective to develop a novel construction whereby 1D SSQCs emerge on the boundary of a lattice in 2D hyperbolic space.

Let $\Pi$ be a (possibly infinite) string of two letters, $\alpha$ and $\beta$, and let $s_{\alpha}(\alpha, \beta)$ and $s_{\beta}(\alpha, \beta)$ be two finite strings of $\alpha$ 's and $\beta$ 's. We can act on $\Pi$ with the inflation rule

$$
\tau:(\alpha, \beta) \mapsto\left(s_{\alpha}(\alpha, \beta), s_{\beta}(\alpha, \beta)\right),
$$

which replaces each $\alpha$ or $\beta$ in $\Pi$ by the string $s_{\alpha}(\alpha, \beta)$ or $s_{\beta}(\alpha, \beta)$, respectively, to obtain a new string $\Pi^{\prime}$. The inverse map is the corresponding deflation rule: 


$$
\tau^{-1}:\left(s_{\alpha}(\alpha, \beta), s_{\beta}(\alpha, \beta)\right) \mapsto(\alpha, \beta) .
$$

Note that, while the inflation rule has a well-defined action on any string $\Pi$ of $\alpha$ 's and $\beta$ 's, the corresponding deflation map only has a well-defined action on a string $\Pi$ that may be uniquely partitioned into substrings of the form $s_{\alpha}(\alpha, \beta)$ and $s_{\beta}(\alpha, \beta)$.

Any inflation rule $\tau$ induces a matrix $M_{\tau}$ that encodes the growth of $N_{\alpha}$ and $N_{\beta}$ (the number of $\alpha$ 's and $\beta$ 's, respectively, in the string). For example, under $\tau:(\alpha, \beta) \mapsto$ $(\alpha \beta \alpha, \alpha \beta \alpha \beta \alpha)$, which corresponds to inflation rule $3 b$ in Table 1 of Ref. [36], we have

$$
\left(\begin{array}{c}
N_{\alpha}^{\prime} \\
N_{\beta}^{\prime}
\end{array}\right)=M_{\tau}\left(\begin{array}{l}
N_{\alpha} \\
N_{\beta}
\end{array}\right), \quad M_{\tau}=\left(\begin{array}{ll}
2 & 3 \\
1 & 2
\end{array}\right) .
$$

If $\lambda$ is the largest eigenvalue of $M_{\tau}(\lambda=3+\sqrt{2})$, we can represent $\Pi$ geometrically as a sequence of $1 \mathrm{D}$ line segments or "tiles" of length $L_{\alpha}$ and $L_{\beta}$, where $\left(L_{\alpha}, L_{\beta}\right)$ is the corresponding left eigenvector of $M_{\tau}$. If $N_{k}^{\alpha}$ and $N_{k}^{\beta}$ denote the number of $\alpha$ 's and $\beta$ 's after $k$ successive applications of $\tau$ to some finite initial string, then in the limit $k \rightarrow \infty$, $\left(N_{k}^{\alpha}, N_{k}^{\beta}\right)$ is the corresponding right eigenvector of $M_{\tau}$ and it grows like $\left(N_{k+1}^{\alpha}, N_{k+1}^{\beta}\right)=\lambda\left(N_{k}^{\alpha}, N_{k}^{\beta}\right)$.

Two strings $\Pi$ and $\Pi^{\prime}$ are locally isomorphic (or locally indistinguishable) if every finite substring contained in one is also contained in the other, so it would be impossible to distinguish them by inspecting any finite segment.

We say that $\Pi$ is a $\tau$ quasicrystal if it is equipped with an inflation rule $\tau$ acting on the two interval types $\alpha$ and $\beta$ [39] such that (i) the matrix $M_{\tau}$ has determinant \pm 1 , with its largest eigenvalue $\lambda$ given by an irrational PisotVijayaraghavan (PV) number, and (ii) the $k$-fold deflation map $\tau^{-k}$ is well defined on $\Pi$ for all positive integers $k$. Condition (i) ensures that $\Pi$ is quasiperiodic and crystalline (in the sense that the Fourier representation of its density profile exhibits delta function diffraction peaks like a crystal), and has $\tau^{-1}$ as the unique deflation rule that inverts $\tau[33,40]$. Condition (ii) implies that a particular $\tau$ quasicrystal is locally isomorphic to every other $\tau$ quasicrystal and, in particular, is locally isomorphic to its own descendants under inflation. In this sense, every $\tau$ quasicrystal is self-similar under inflation by $\tau$. If a $\tau$ quasicrystal $\Pi$ is mapped to itself by ( $k$ successive applications of) $\tau$, we say it is ( $k$-fold) self-same, an even stronger scaleinvariance property than self-similarity.

\section{UNIT-CELL ASSIGNMENT ON THE HYPERBOLIC DISK}

A regular $\{p, q\}$ tessellation is constructed from regular $p$-sided polygons (with $p$ geodesic edges), where $q$ such polygons meet at each vertex. Such a tessellation exists for any $p, q>2$ : When $(1 / p)+(1 / q)$ is greater than, equal to, or less than $1 / 2$, respectively, it is a tessellation of the sphere, Euclidean plane, or hyperbolic plane $[28,41]$.

Consider a regular $\{p, q\}$ tessellation of the hyperbolic plane, $(1 / p)+(1 / q)<\frac{1}{2}$. In this paper, we focus on the case where $p$ and $q$ are both finite. Let $S$ (the "seed") be a finite, simply connected patch of tiles, and let it also be convex, which, here, means that, if $\theta_{c}$ is the interior angle of $S$ at a vertex $c$, then $\theta_{c}<2 \pi(1-1 / q)$ for each boundary vertex (when $q>3$ ) or $\theta_{a}+\theta_{b}<4 \pi(1-1 / q$ ) for all nearest-neighbor pairs $\langle a, b\rangle$ of boundary vertices (when $q=3$ ). On the boundary of $S$, we can assign different unitcell types between which the d.o.f. live. The assignment procedure, illustrated in Fig. 2(a), is the following. Consider the union $U(S)$ of all tiles that share a vertex with the boundary of $S$ (excluding the tiles in $S$ itself). In the interior of $U(S)$, we can draw a curve that crosses every tile of $U(S)$ exactly once. This curve partitions every tile into two pieces: one contained in the interior of the curve and one in the exterior. If the interior part of the tile has $n$ vertices, then we call it a type- $n$ unit cell. Once every cell on the boundary of $S$ has been labeled in this manner, we can write down a corresponding string $Q(S)$ of cell types as in Fig. 2(c). These strings are inside square brackets to emphasize that they are cyclically ordered. When $S$ is convex, only two cell types appear on its boundary (type 1 and type 2 when $q>3$, and type 2 and type 3 when $q=3$ ), but since the seed $S$ in Fig. 2(a) is not convex, a type-4 cell also appears.

In a concrete holographic TN model, a tensor is placed on each vertex of $S$, and contractions are performed between nearest-neighbor tensors along the tessellation edges. Extra tensors can be added to the edges, as in the
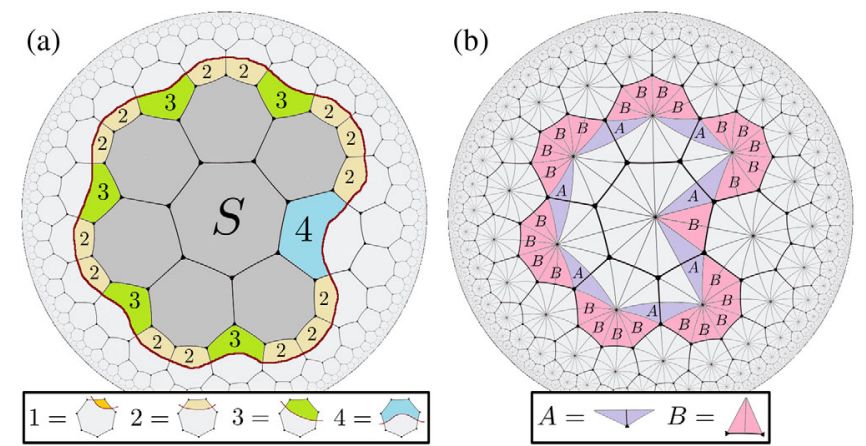

(c)

$Q(S)=[42223223223223223222]$

$\Lambda(S)=[B A B A B B B B B A B B B B A B B B B A B B B B A B B B B A B B B B]$ (d)

$$
2 \leftrightarrow B \quad 3 \leftrightarrow B A B \quad 4 \leftrightarrow B A B A B \quad \cdots \quad n \leftrightarrow B \underbrace{A B \ldots A B}_{n-2 \text { times }}
$$

FIG. 2. Illustration of unit-cell and letter assignment on the boundary of a simply connected tile set $S$, in the $\{7,3\}$ tiling. (a) Unit-cell assignment. (b) Letter assignment. (c) Cyclically ordered strings of unit cells and letters. (d) Correspondence between unit cells and letters in the $\{7,3\}$ case. 
hyperinvariant networks of Evenbly [21], or bulk ancilla d.o.f. to the vertex tensors, as in the holographic quantum error correcting codes of Ref. [16]. The boundary d.o.f. are defined on legs placed on the edges of the tessellation emanating from $S$, which are cut by the partitioning curve in Fig. 2(a). Thus, the distance in the network between two adjacent d.o.f. on the boundary of $S$ depends on the type of unit cell between them. In this sense, the cell types defined here behave analogously to the interval types $L_{\alpha}$ and $L_{\beta}$ between d.o.f. placed on the vertices of a $d=1$ quasicrystal.

There is an alternative way to label the boundary of $S$ using only two symbols, which treats the $q=3$ and $q>3$ cases more uniformly and continues to apply even when $S$ is nonconvex. Decompose every tile into the fundamental domains of the tiling symmetry group, which are right triangles of angles $\pi / 2, \pi / q$, and $\pi / p$. This decomposition is shown in Fig. 2(b). Now, consider the set of such right triangles in the interior of $S$ that share a vertex with the boundary of $S$. Within this set, we identify and label two types of isosceles triangles- $A$ and $B$. Each $A$ shares one vertex and no edges with the boundary of $S$, and each $B$ shares two vertices and one edge [see Fig. 2(b)]. This procedure, called letter assignment, produces a cyclically ordered string $\Lambda(S)$, distinct from $Q(S)$, containing only $A$ 's and $B$ 's [see Fig. 2(c)].

There is a way to extract $Q(S)$ from $\Lambda(S)$ and vice versa by mapping each type- $n$ unit cell to a string of $A$ 's and $B$ 's. This correspondence is

$$
\begin{aligned}
1 & \leftrightarrow A^{-1} \\
2 & \leftrightarrow A^{q-3 / 2} B A^{q-3 / 2} \\
3 & \leftrightarrow A^{q-3 / 2} B A^{q-2} B A^{q-3 / 2} \\
& \vdots \\
n & \leftrightarrow A^{q-3 / 2}\left(B A^{q-2}\right)^{n-2} B A^{q-3 / 2},
\end{aligned}
$$

where $n \leq p$. Here, if $f(A, B)$ is any finite substring, then $(f(A, B))^{x}$ denotes its $x$-fold repetition whenever $x \in \mathbb{Z}_{\geq 0}$, with $(f(A, B))^{0}$ the empty substring. Fractional and negative exponents behave in the usual way, e.g., $A^{x} A^{-y}=$ $A^{x-y}$ for all $x, y \in \mathbb{Q}$.

\section{INFLATION RULES ON THE HYPERBOLIC DISK}

We can append the ring of tiles $U(S)$ to the set $S$ to obtain a new larger set $S^{\prime}=S+U(S)$, as in Fig. 3. Accordingly, we add a layer of tensors to our TN to obtain a holographic TN model on $S^{\prime}$ whose d.o.f. are defined between the unit cells on the boundary of $S^{\prime}$. The vertices that were formerly on the boundary (i.e., the ones that were not completely surrounded by the tiles of $S$ ) are now in the interior (i.e., are completely surrounded by the tiles of $S^{\prime}$ ). We dub this procedure "vertex completion."

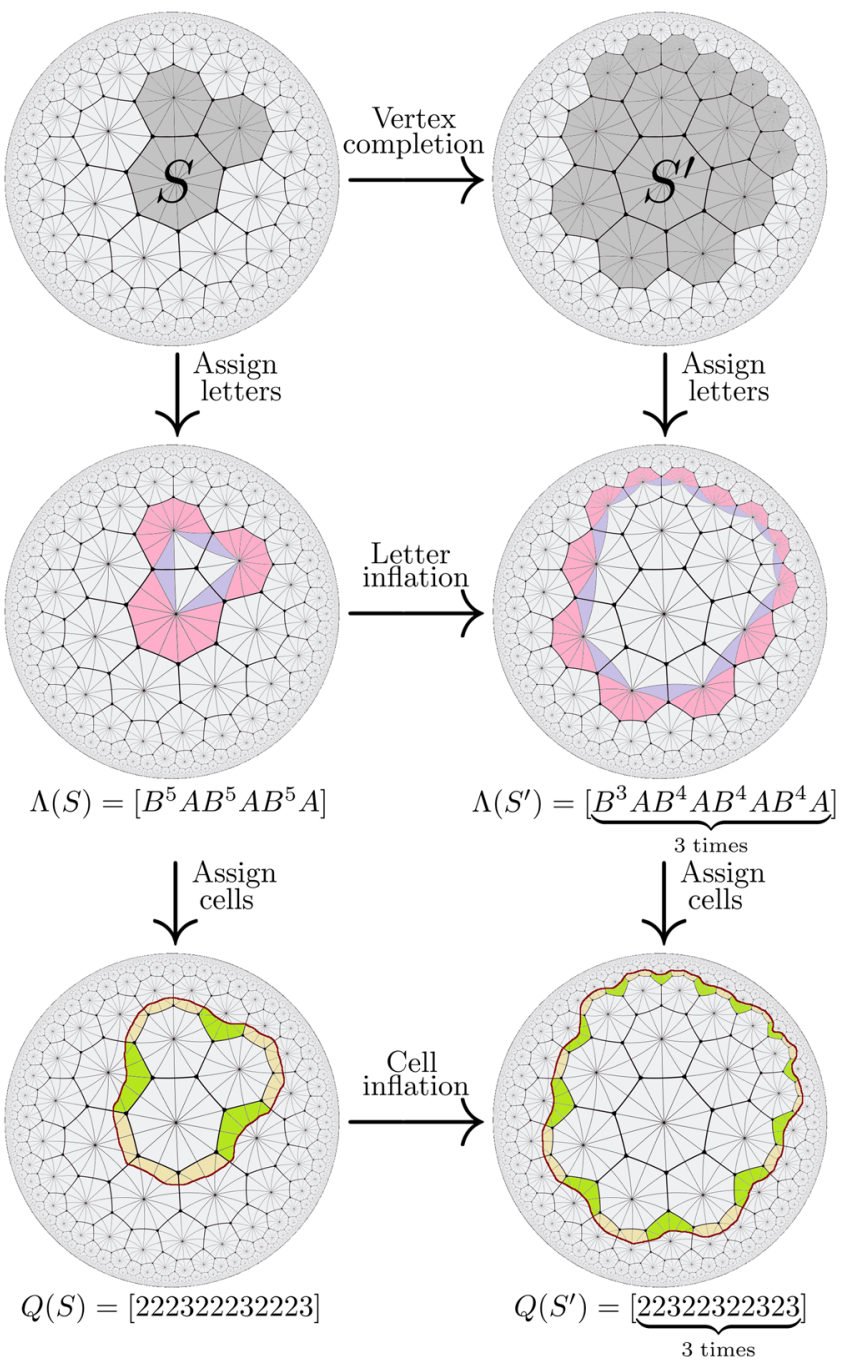

FIG. 3. Demonstration of how vertex completion induces inflation rules on boundary letters and unit cells, for the $\{7,3\}$ tiling. The induced letter inflation rule $(A, B) \mapsto\left(A^{-1} B^{-5}, B^{4} A\right)$ is the same for every tile set $S$, convex or not. When $S$ is convex, only type-2 and type- 3 unit cells appear, and there is an induced unit-cell inflation rule $(2,3) \mapsto(223,23)$. Note that vertex completion preserves convexity and that every tile set $S$ becomes convex after finitely many vertex completions.

Vertex completion induces a mapping $\tau_{\Lambda}(p, q): \Lambda(S) \rightarrow$ $\Lambda\left(S^{\prime}\right)$. Remarkably, $\tau_{\Lambda}(p, q)$ is an inflation rule on $A$ and $B$ and is the same for every $S$. If we define the stringless

$$
s_{L}:=A^{1 / 2} B^{(p-2) / 2}, \quad s_{R}:=B^{(p-2) / 2} A^{1 / 2},
$$

then $\tau_{\Lambda}(p, q)$ can be written

$$
\begin{gathered}
A \mapsto\left(s_{L} s_{R}\right)^{-1}=A^{-1 / 2} B^{2-p} A^{-1 / 2}, \\
B \mapsto \begin{cases}s_{L}\left(s_{R} s_{L}\right)^{q-3 / 2} B^{-1}\left(s_{R} s_{L}\right)^{q-3 / 2} s_{R} & (q \text { odd }) \\
\left(s_{R} s_{L}\right)^{q-2 / 2} B^{-1}\left(s_{R} s_{L}\right)^{q-2 / 2} & (q \text { even }) .\end{cases}
\end{gathered}
$$


This version of the substitution rule is the canonical one, in the sense that it respects the symmetry of the parent tile $(A$ or $B$ ) under reflection about its midline, but another equivalent version (which is asymmetric) is simpler and more convenient:

$$
A \mapsto A^{-1} B^{2-p}, \quad B \mapsto B^{p-3} A\left(B^{p-2} A\right)^{q-3} .
$$

Note that two substitution rules $\{\alpha, \beta\} \mapsto\left\{s_{\alpha}, s_{\beta}\right\}$ and $\{\alpha, \beta\} \mapsto\left\{\bar{s}_{\alpha}, \bar{s}_{\beta}\right\}$ are equivalent if $\bar{s}_{\alpha}=u s_{\alpha} u^{-1}$ and $\bar{s}_{\beta}=$ $u s_{\beta} u^{-1}$ for some finite string $u=u(\alpha, \beta)$. In particular, the two substitution rules (5) and (6) both correspond to the substitution matrix

$$
M_{\tau_{\Lambda}(p, q)}=\left(\begin{array}{cc}
-1 & q-2 \\
2-p & (p-2)(q-2)-1
\end{array}\right) .
$$

The corresponding induced map $\tau_{Q}(p, q): Q(S) \rightarrow$ $Q\left(S^{\prime}\right)$ is also an inflation rule on two unit-cell types whenever $S$ is convex. The rule $\tau_{Q}(p, q)$ can be extracted by combining $\tau_{\Lambda}(p, q)$ with the cell-to-letter mapping. When $q>3$,

$$
\begin{aligned}
1 & \mapsto 1^{q-4 / 2}\left(21^{q-3}\right)^{p-3} 21^{q-4 / 2}, \\
2 & \mapsto 1^{q-4 / 2}\left(21^{q-3}\right)^{p-4} 21^{q-4 / 2},
\end{aligned}
$$

and when $q=3$,

$$
\begin{aligned}
2 & \mapsto 3^{\frac{1}{2}} 2^{p-5} 3^{\frac{1}{2}}, \\
3 & \mapsto 3^{\frac{1}{2}} 2^{p-6} 3^{\frac{1}{2}} .
\end{aligned}
$$

The matrices $M_{\tau_{Q}(p, q)}$ and $M_{\tau_{\Lambda}(p, q)}$ are related by a change of basis. They have unit determinant and eigenvalues

$$
\lambda_{ \pm}(p, q)=\gamma_{p, q} \pm\left(\gamma_{p, q}^{2}-1\right)^{1 / 2}
$$

with

$$
\gamma_{p, q}:=\frac{(p-2)(q-2)}{2}-1
$$

These results are consistent with the previous findings of Ref. [42]. Note that the largest eigenvalue $\lambda_{+}(p, q)$ is irrational and PV whenever $(1 / p)+(1 / q)<\frac{1}{2}$. When $S$ is nonconvex, $\tau_{Q}(p, q)$ acts on the many cell types of $Q(S)$ in a complicated way. However, every tile set $S$ maps to a convex set under finitely many vertex completions, and vertex completion preserves convexity once established.

\section{CONFORMAL QUASICRYSTALS ON THE BOUNDARY OF THE DISK}

Consider any finite, simply connected tile set $S$ of the $\{p, q\}$ tessellation. By repeatedly carrying out layers of vertex completions, we generate a sequence of tile sets $\left\{S_{n}\right\}_{n \in \mathbb{Z}_{>0}}$, with initial element $S_{0}=S$, and $\left\{Q\left(S_{n}\right)\right\}_{n \in \mathbb{Z}_{>0}}$. Concurrently, we can consider a family of holographic TN models defined on each $S_{n}$ whose d.o.f. live on the boundary of $S_{n}$. In the limit $n \rightarrow \infty$, the tile set covers the entire hyperbolic disk, and we can ask about the unitcell assignment $Q_{\infty}(S):=\lim _{n \rightarrow \infty} Q\left(S_{n}\right)$ living at the disk boundary. We can interpret $Q_{\infty}(S)$ as a type of emergent quasicrystal harboring the d.o.f. To see this case, recall that for all $n$ above some $n^{*}>0, Q\left(S_{n}\right)$ contains only two cell types, and thus $\tau_{Q}(p, q)$ acts on these two cell types as an inflation rule. Taking $Q\left(S_{n^{*}}\right)$ as an initial string and iterating $\tau_{Q}(p, q)$ infinitely many times generates the infinite, cyclically ordered string $Q_{\infty}(S)$, which is selfsame under $\tau_{Q}(p, q)$. Thus, $Q_{\infty}(S)$ is locally isomorphic to a $\tau_{Q}(p, q)$ quasicrystal, which is the promised quasicrystalline interpretation. We call any $Q_{\infty}(S)$ obtained in this manner a $\{p, q\}$ conformal quasicrystal (CQC).

\section{DISCRETE CONFORMAL GEOMETRY}

Our construction naturally endows CQCs with a discrete analog of conformal geometry. In the continuum, conformal geometry refers to those properties of a (pseudo-) Riemannian manifold that are invariant under positiondependent rescalings (or Weyl transformations) of the metric tensor $g_{\mu \nu}(x) \mapsto \Omega^{2}(x) g_{\mu \nu}(x)$. Thus, length scales are not well defined in conformal geometry. Conformal properties are preserved under conformal maps, diffeomorphisms fixing the metric up to a Weyl transformation. These include all the diffeomorphisms in $d=1$ and just the angle-preserving diffeomorphisms in $d \geq 2$. In physics, conformal geometries underlie conformal field theories (CFTs), which are quantum field theories that are symmetric under such conformal maps $[43,44]$. Here, we show that one can relate any two CQCs with the same $\{p, q\}$ via a discrete analog of a Weyl transformation. Hence, CQCs inherit discrete conformal geometry, defined to be the properties fixed under discrete Weyl transformations.

Let $M(S) \neq S$ denote a finite, simply connected tile set obtained from $S$ in any manner. Here, $Q_{\infty}(S)$ and $Q_{\infty}(M(S))$ are locally isomorphic, but they differ in their global structures. To relate $Q_{\infty}(S)$ and $Q_{\infty}(M(S))$, a map $\Omega$ such that $\Omega\left(Q_{\infty}(S)\right)=Q_{\infty}(M(S))$ is needed. We say that any such $\Omega$ is a "discrete Weyl transformation." Here, $\Omega$ acts upon the global structure of the CQC by a positiondependent set of inflations and deflations. The TN model is correspondingly transformed by adjoining or removing tensors from the edges in a position-dependent way.

The intuition behind our definition comes from an analogy with Weyl transformations in continuum 
AdS/CFT. There, a Weyl transformation corresponds to a change of choice of how to approach the boundary [3]. More precisely, the line element for $(d+1)$-dimensional hyperbolic space can be written in global coordinates as

$$
d s^{2}=\frac{d \rho^{2}+\sin ^{2}(\rho) d \Omega_{d}^{2}}{\cos ^{2}(\rho)}
$$

where $\rho \in[0, \pi / 2)$ is the radial coordinate and $d \Omega_{d}^{2}$ is the line element on the unit $d$ sphere $S_{d}$. Now, consider a family of $d$-dimensional hypersurfaces of spherical topology, parametrized by $\rho(x, \epsilon)=(\pi / 2)-\epsilon f(x)$, where $x$ are the coordinates on $S_{d}$ and $f(x)$ is an arbitrary positive function on $S_{d}$. To approach the boundary, we take $\epsilon \rightarrow 0^{+}$ in an $x$-independent way. As $\epsilon \rightarrow 0^{+}$, a constant- $\epsilon$ hypersurface has an induced line element:

$$
d s_{\epsilon}^{2}(x) \sim \frac{2}{\epsilon^{2}} \frac{d \Omega_{d}^{2}(x)}{f^{2}(x)} .
$$

Thus, we see that changing the hypersurface profile $f(x) \mapsto \Omega^{-1}(x) f(x)$ induces the Weyl transformation

$$
d s_{\epsilon}^{2}(x) \mapsto \Omega^{2}(x) d s_{\epsilon}^{2}(x) .
$$

In our construction, the choice of initial tile set $S$ is analogous to the choice of hypersurface profile $f(x)$, while iterating vertex completions infinitely many times corresponds to taking $\epsilon \rightarrow 0^{+}$. Thus, it is natural to interpret $S \mapsto$ $M(S)$ as inducing a Weyl-like transformation $Q_{\infty}(S) \mapsto$ $\Omega\left(Q_{\infty}(S)\right)=Q_{\infty}(M(S))$.

\section{SYMMETRIES OF CONFORMAL QUASICRYSTALS IN $\boldsymbol{d}=\mathbf{1}$}

Now, let us emphasize two different types of symmetry possessed by any CQC $Q_{\infty}(S)$ of type $\{p, q\}$. First, it has a kind of exact scale symmetry: invariance under $\tau_{Q}(p, q)$. Second, it is invariant, up to a discrete Weyl transformation, under an infinite discrete group called the triangle group, $\Delta(2, p, q)$. This group is the symmetry group of the $\{p, q\}$ tiling; it is generated by reflections across the fundamental right-triangle domains of the tiling [45]. Each such reflection induces a conformal map of the boundary into itself. Under an element of $\Delta(2, p, q), S$ maps to a different simply connected $S^{\prime}$, which produces $Q_{\infty}\left(S^{\prime}\right)$. Since $S^{\prime}=$ $M(S)$ for some $M$, it follows that $Q_{\infty}(S)$ and $Q_{\infty}\left(S^{\prime}\right)$ are related by a discrete Weyl transformation, as required. Thus, we regard $\Delta(2, p, q)$ as a discretization (i) of the isometry group of the hyperbolic disk that acts on the bulk tessellation and (ii) of the conformal group of the circular boundary that acts on the CQCs.

\section{DISCUSSION}

Before discussing our results, let us briefly summarize our findings.

The past decade has seen exciting developments in our understanding of quantum gravity: In particular, arguments based on holography and gauge-gravity duality have led to a tantalizing but still-fragmentary picture in which spacetime emerges from the pattern of entanglement in an underlying quantum system. In particular, there has been much recent interest in attempts to make this picture more concrete, in the context of AdS/CFT, by developing a discrete formulation of holography, based on replacing the continuous hyperbolic "bulk" space by a discrete regular tessellation of hyperbolic space (or some other tessellation that respects a large discrete subgroup of the original space's symmetries). These developments are part of a family of ideas that are sometimes summarized by the slogan "it from qubit".

Previous works have focused on discretizing the bulk geometry, but they have not thought through the corresponding implications for the boundary geometry. In this paper, we have shown that when one discretizes the bulk geometry in a natural way (e.g., on a regular tessellation), one also induces a remarkable discretization of the lowerdimensional "boundary" geometry into a fascinating new kind of discrete geometric structure, which we call a "conformal quasicrystal." The main goals of this paper were to point out the existence of these new structures, to define and explain their basic properties, and to emphasize that they appear to be the natural discrete spaces living on the boundary side of the holographic duality.

We think these observations provide important clues for the ongoing efforts to formulate a discrete version of holography (perhaps in terms of tensor networks) [46]: In a correct discrete formulation of AdS/CFT, we expect the boundary theory to live on a conformal quasicrystal (rather than on an ordinary lattice, as imagined in previous works). Similarly, since the boundary theory in AdS/CFT is scale invariant, it suggests that the natural way to discretize and numerically simulate scale-invariant systems (such as conformal field theories, or condensed matter systems near their critical points) is to discretize them on a conformal quasicrystal, rather than on a periodic lattice. Thus, we hope that this work opens the door to more efficient simulation of the dynamics and quantum states of such systems (although much work remains in order to translate this hope into practice).

Let us now mention various directions for future work. We expect our definitions and results to extend readily to higher dimensions. Consider, for example, the self-dual $\{3,5,3\}$ regular honeycomb in three-dimensional hyperbolic space. This honeycomb is constructed by gluing together icosahedra such that the vertex figure at every corner is a dodecahedron [28,41]. Now, imagine that we take the initial seed $S$ to be one such icosahedron and then 
carry out vertex completion, assigning boundary unit cells at every step. At each step, we obtain a 2D layer of spherical topology with 12 points of exact fivefold orientational symmetry (or, more precisely, $D_{5}$ symmetry), lying radially above the 12 vertices of the initial icosahedron. Hence, after iterating infinitely many times, we obtain a layer of spherical topology at the boundary, tesselated by an infinite number of tiles. This layer still has 12 points of $D_{5}$ symmetry, and in the vicinity of any such point, it appears to be an infinite tiling of the 2D Euclidean plane. Since points of $D_{5}$ symmetry are forbidden in an ordinary (periodic) 2D crystal, we expect the boundary to once again be quasicrystalline. Indeed, the $2 \mathrm{D}$ quasicrystalline tilings with fivefold orientational order are all closely related to the Penrose tiling [33,35,37,38,49]. (Note that, although the usual Penrose tiling has, at most, one point of exact $D_{5}$ symmetry, the Penrose-like CQC living at the boundary of $\{3,5,3\}$ can have 12 such points, as explained above. This case is the higher-dimensional analogue of a phenomenon that already occurs in the 1D CQCs constructed above: The CQC generated from a regular $p$ gon initial seed will have $2 p$ points of perfect reflection symmetry, in contrast to the self-similar 1D quasicrystal that it locally resembles, which has, at most, one such point.)

The conjecture that $\{3,5,3\}$ hosts a CQC generalization of the Penrose tiling on its boundary is supported by a calculation [50] of the ratio $N_{k+1} / N_{k}$ as $k \rightarrow \infty$, where $N_{k}$ is the number of boundary cells after $k$ layers of vertex completion (this ratio is expected to be equal to the largest eigenvalue of the matrix $M_{\tau}$ associated with the inflation rule $\tau$ induced by the vertex completion). The limiting ratio is found to be $N_{k+1} / N_{k} \rightarrow \varphi^{8}$, where $\varphi$ is the golden ratio. Note that this result is irrational and PV (as expected for a $2 \mathrm{D}$ quasicrystal) and is a power of $\varphi$ (as expected for a quasicrystal with fivefold orientational order, in particular $[33,35,37,38])$. If we compare this result to the scaling ratio for the standard Penrose tiling $\left(N_{k+1} / N_{k} \rightarrow \varphi^{2}\right)$, it suggests either that a single layer of vertex completion in the $\{3,5,3\}$ honeycomb corresponds to four iterations of the usual Penrose tiling inflation rule or that the Penrose tiling and $\{3,5,3\}$ CQC are related in some way besides local isomorphism.

We have seen that applying vertex completion to a regular $\{p, q\}$ tiling of hyperbolic space yields a $\tau_{Q}(p, q)$ quasicrystal. There are, however, other $\tau$ quasicrystals that are not obtained in this way-like the example given above in Eq. (3). We wonder whether every remaining inflation rule $\tau$ is also naturally induced by applying some appropriate generalization of vertex completion to some appropriate tiling of hyperbolic space. Tilings beyond the regular $\{p, q\}$ type seem to be necessary.

The remarkable conformal geometry of CQCs suggests that their importance extends beyond the realm of holographic TN models. We propose to use them as the underlying spaces on which to discretize CFTs, in at least two ways. First, one can discretize a QFT partition function onto a $\tau$ quasicrystal directly. After implementing a real-space renormalization-group procedure whereby d.o.f. are decimated according to $\tau^{-1}$, the renormalization-group fixed points can be identified with the discrete CFTs. Second, one can work, by analogy, with AdS/CFT. Consider the partition function $Z_{S, n}[y]$ of a discretized field theory on the $n$-fold image $S_{n}$ under vertex completion of a simply connected subset $S$ of the bulk tessellation, such that the fields take values $y$ at the boundary of $S_{n}$. As $n \rightarrow \infty$, we propose to interpret $Z_{S, \infty}[y]$ as the moment-generating function of a discrete CFT on the boundary.

From a practical standpoint, such CQC-based discretizations may be important for studying scale-invariant physical systems such as CFTs or condensed matter systems at their critical points. These discretizations should permit finite-size simulations of such systems, in ways that preserve an exact discrete subgroup of their scale symmetry. This case is in contrast with ordinary lattice gauge theory simulations or other periodic lattice models, which instead preserve an exact discrete subgroup of translation symmetry, at the cost of breaking scale invariance. We can attempt to finitize a numerical calculation on a conformal quasicrystal by restricting ourselves to an annulus in the reciprocal space of the quasicrystal and imposing the boundary condition that observables on the inner and outer boundaries of the annulus are the same up to an appropriate scale factor. This method would provide the analog in discrete CFTs of a periodic boundary condition in a periodic lattice model, along the scale direction instead of in real space. The resulting finite problem can then be attacked with a large arsenal of Monte Carlo and tensor network methods. Our hope is that this approach may lead to more accurate and efficient numerical simulation of such systems, perhaps allowing us to simulate much larger systems and leading to qualitative improvements in our understanding.

TN algorithms based on inflations and deflations may also find use in the numerical description of quantum critical states. We can imagine implementing a MERAlike coarse-graining scheme induced by the quasicrystalline structure. Consider the simplest $\tau$ quasicrystals, the 1D Fibonacci quasicrystals generated by the inflation rule $\tau:\{\alpha, \beta\} \mapsto\left\{\beta^{1 / 2} \beta^{1 / 2}, \beta^{1 / 2} \alpha \beta^{1 / 2}\right\}$. Given an initial Fibonacci quasicrystal $\Pi$, we can use a deflationlike rule to obtain a coarser quasicrystal $c(\Pi)$ in two steps: (i) Slice every $\beta$ interval into two halves, $\beta^{1 / 2}$, resulting in a string that can be uniquely partitioned into substrings of the form $\beta^{1 / 2} \beta^{1 / 2}$ and $\beta^{1 / 2} \alpha \beta^{1 / 2}$; (ii) glue these substrings together to form intervals $\alpha^{\prime}=\beta^{1 / 2} \beta^{1 / 2}$ and $\beta^{\prime}=\beta^{1 / 2} \alpha \beta^{1 / 2}$ of respective lengths $L_{\alpha^{\prime}}=L_{\beta}$ and $L_{\beta^{\prime}}=L_{\alpha}+L_{\beta}$. Iterating this procedure yields a sequence $\left\{c^{k}(\Pi)\right\}_{k \in \mathbb{Z}_{\geq 0}}$ of successively coarser quasicrystals. We can now construct a MERA-like circuit as follows. Embed each quasicrystal $c^{k}(\Pi)$ into the two-dimensional plane, such that $c^{k+1}(\Pi)$ is parallel to and 


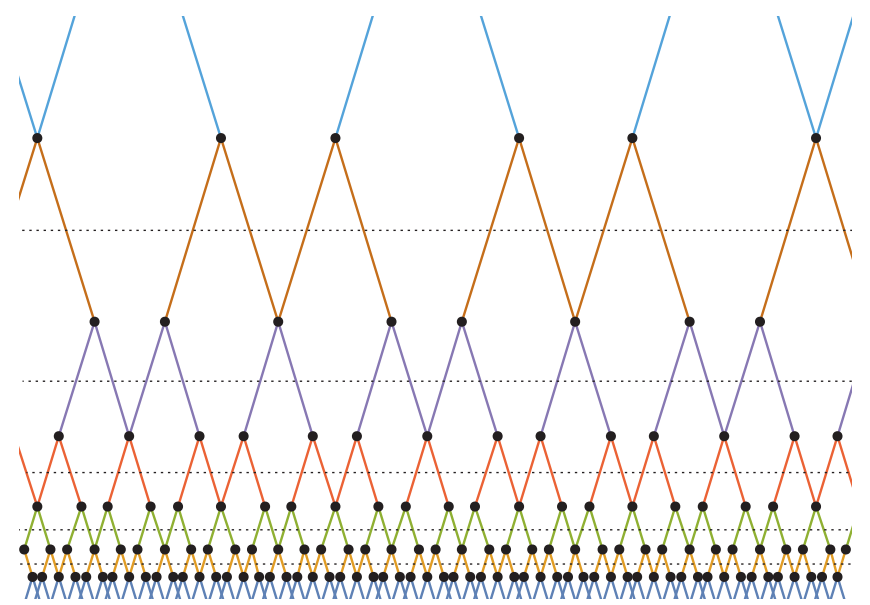

FIG. 4. A "quasi-MERA" tensor network based on the 1D Fibonacci quasicrystal. Vertices denote tensors, and edges denote contractions between tensors. Three- and four-legged tensors represent isometries and disentanglers, respectively. Dotted lines separate layers related by the Fibonacci deflation rule.

lies above $c^{k}(\Pi)$ for every $k$. Assign to every point in $c^{k}(\Pi)$ a tensor, and contract each tensor only with its nearest neighbors in $c^{k+1}(\Pi)$ and $c^{k-1}(\Pi)$. This method results in three- and four-legged tensors; if we choose these to be isometries and unitary disentanglers, respectively, then we acquire the desired MERA-like circuit. We call this network, depicted in Fig. 4, the "quasi-MERA" due to its relationship with the quasicrystalline inflation rule $\tau$.

To see why the quasi-MERA may be special, we contrast the deflation-based decimation procedure of the $\tau$ quasicrystal with the standard block decimation of the periodic crystal. Unlike the periodic crystal, for every $\tau$-quasicrystal layer, there is a local refinement (inflation) rule that is inverted by an unambiguous local coarse-graining (deflation) rule. To see why this cannot hold for an ordinary crystal, consider the example of a 1D periodic lattice with intervals of length $a$. We can produce a more refined lattice (with intervals of length $a / 2$ ) by the local rule of chopping each interval in half, but the inverse coarse-graining transformation is not locally well defined: There is an ambiguity about which pair of short intervals we need to glue together to make a long one, so $N$ "joiners" at widely separated points on the lattice would make different, incompatible choices about which tiles to join, and $\mathcal{O}(N)$ defects would be produced. Hence, block decimating a periodic lattice destroys information about how to locally recover the original lattice. Since the ordinary (binary) MERA architecture relies on this form of block decimation, its description of a quantum state is necessarily lossy, even at criticality. By contrast, when coarse-graining the Fibonacci quasicrystal as in Fig. 4, one can locally and unambiguously determine, from the tiles $\alpha$ and $\beta$ in a given layer, where to place the larger intervals $\alpha^{\prime}$ and $\beta^{\prime}$ in the next layer up. In this sense, the coarse-graining transformation for a $\tau$ quasicrystal is lossless, in contrast to the block decimation of a periodic lattice. For this reason, we ask whether the quasi-MERA can give an exact description of a quantum critical state provided that the system is already discretized on the appropriate quasicrystal. This is a nontrivial statement that requires numerical checking and benchmarking.

Geometrically, the quasi-MERA construction of Fig. 4 generates a novel emergent "tiling" of the hyperbolic halfplane by hexagons, with three or four glued together at a vertex. However, this network should be conceptually separated from the TNs considered in the main paper because, unlike those TNs, this network can harbor unitary disentanglers and has a preferred causal direction. Specifically, we do not believe that the quasi-MERA should be interpreted as a discretization of a spacelike slice of $\mathrm{AdS}_{2+1}$. In view of Refs. [18] and [27], we ask whether Fig. 4 could be interpreted as a discretization of the kinematic space or some other causal geometry.

We leave the investigation of these many exciting possibilities for future studies.

\section{ACKNOWLEDGMENTS}

We thank Ehud Altman, Guifre Vidal, and Mudassir Moosa for helpful discussions. L. B. acknowledges support from a NSERC Discovery Grant. M. D. acknowledges the National Science Foundation and the Perimeter Institute for Theoretical Physics for partial funding. F. F. acknowledges support from the English Speaking Union and New College, Oxford. Research at the Perimeter Institute is supported by the Government of Canada through the Department of Innovation, Science and Economic Development, and by the Province of Ontario through the Ministry of Research and Innovation. Figures 2 and 3 have been generated with the assistance of the KaleidoTile software [51].

[1] J. M. Maldacena, The Large N Limit of Superconformal Field Theories and Supergravity, Int. J. Theor. Phys. 38, 1113 (1999); [Adv. Theor. Math. Phys. 2, 231 (1998)].

[2] E. Witten, Anti-de Sitter Space and Holography, Adv. Theor. Math. Phys. 2, 253 (1998).

[3] J. Kaplan, Lectures on Ads/CFT from the Bottom Up, Johns Hopkins University course notes (to be published).

[4] M. Van Raamsdonk, Building Up Spacetime with Quantum Entanglement, Gen. Relativ. Gravit. 42, 2323 (2010); [Int. J. Mod. Phys. D 19, 2429 (2010)].

[5] K. G. Wilson, Confinement of Quarks, Phys. Rev. D 10, 2445 (1974).

[6] G. Vidal, Entanglement Renormalization, Phys. Rev. Lett. 99, 220405 (2007).

[7] G. Vidal, Class of Quantum Many-Body States that Can Be Efficiently Simulated, Phys. Rev. Lett. 101, 110501 (2008). 
[8] G. Vidal, Entanglement Renormalization: An Introduction, in Understanding Quantum Phase Transitions (CRC Press, New York, 2010).

[9] R. N. C. Pfeifer, G. Evenbly, and G. Vidal, Entanglement Renormalization, Scale Invariance, and Quantum Criticality, Phys. Rev. A 79, 040301(R) (2009).

[10] G. Evenbly and G. Vidal, Tensor Network States and Geometry, J. Stat. Phys. 145, 891 (2011).

[11] R. Orus, Advances on Tensor Network Theory: Symmetries, Fermions, Entanglement, and Holography, Eur. Phys. J. B 87, 280 (2014).

[12] B. Swingle, Entanglement Renormalization and Holography, Phys. Rev. D 86, 065007 (2012).

[13] B. Swingle, Constructing Holographic Spacetimes Using Entanglement Renormalization, arXiv:1209.3304.

[14] X. L. Qi, Exact Holographic Mapping and Emergent SpaceTime Geometry, arXiv:1309.6282.

[15] J. Molina-Vilaplana, Holographic Geometries of OneDimensional Gapped Quantum Systems from Tensor Network States, J. High Energy Phys. 05 (2013) 024.

[16] F. Pastawski, B. Yoshida, D. Harlow, and J. Preskill, Holographic Quantum Error-Correcting Codes: Toy Models for the Bulk/Boundary Correspondence, J. High Energy Phys. 06 (2015) 149.

[17] P. Hayden, S. Nezami, X. L. Qi, N. Thomas, M. Walter, and Z. Yang, Holographic Duality from Random Tensor Networks, J. High Energy Phys. 11 (2016) 009.

[18] B. Czech, L. Lamprou, S. McCandlish, and J. Sully, Tensor Networks from Kinematic Space, J. High Energy Phys. 07 (2016) 100.

[19] M. Han and L. Y. Hung, Loop Quantum Gravity, Exact Holographic Mapping, and Holographic Entanglement Entropy, Phys. Rev. D 95, 024011 (2017).

[20] X. L. Qi, Z. Yang, and Y.Z. You, Holographic Coherent States from Random Tensor Networks, J. High Energy Phys. 08 (2017) 060.

[21] G. Evenbly, Hyperinvariant Tensor Networks and Holography, Phys. Rev. Lett. 119, 141602 (2017).

[22] T. J. Osborne and D. E. Stiegemann, Dynamics for Holographic Codes, arXiv:1706.08823.

[23] N. Bao, C. Cao, S. M. Carroll, and A. Chatwin-Davies, De Sitter Space as a Tensor Network: Cosmic No-Hair, Complementarity, and Complexity, Phys. Rev. D 96, 123536 (2017).

[24] A. Jahn, M. Gluza, F. Pastawski, and J. Eisert, Holography and Criticality in Matchgate Tensor Networks, Sci. Adv. 5, eaaw0092 (2019).

[25] M. A. Nielsen and I. L. Chuang, Quantum Computation and Quantum Information (Cambridge University Press, Cambridge, England, 2010).

[26] B. Czech, L. Lamprou, S. McCandlish, and J. Sully, Integral Geometry and Holography, J. High Energy Phys. 10 (2015) 175.

[27] A. Milsted and G. Vidal, arXiv:1812.00529.

[28] H. S. M. Coxeter, Regular Honeycombs in Hyperbolic Space, Proc. Intl. Congr. Math. 3, 155 (1954).

[29] Note that, in this paper, as in Refs. [16,21,24], we focus on ordinary regular $\{p, q\}$ tessellations (i.e., tessellations where each tile is a regular $p$-gon, $q$ such tiles meet at each vertex, and $p$ and $q$ are both finite) since these represent the natural discretizations of theories that are local in the bulk. Another fascinating possibility is to relax this locality restriction and consider $\{p, \infty\}$ tilings of the hyperbolic plane in which infinitely many tiles meet at each vertex; in this case, tile edges are infinitely long, and the tile vertices live at infinity, on the boundary of the hyperbolic plane, where they can then induce a periodic structure at infinity. Reference [22] contains a detailed exploration of this latter possibility and, in particular, in this context, presents an interesting toy model in which the discrete symmetries in the bulk may be related to a discretization of the conformal group of the boundary. The dual cases $\{\infty, q\}$ consist of regular polygons with an infinite number of sides (apeirogons), with $q$ meeting at each vertex. When $q$ is a prime $p$, the boundary theory is then naturally described by the $p$-adic numbers [30].

[30] L. Brekke and P. G. O. Freund, p-Adic Numbers in Physics, Phys. Rep. 233, 1 (1993).

[31] Yet another possibility is to replace the regular tiling in the bulk by a random tensor network as in Ref. [17]. In AdS/CFT, a key role is played by the isometry group of the bulk AdS space, and a regular tiling has the advantage that it preserves a large discrete subgroup of this symmetry. However, for some purposes, a random tensor network may also have certain advantages, and it would be interesting to investigate the mathematical structure that it induces on the boundary. If the random tensor network in the bulk respects the properties of the regular tiling in a statistical sense, then the random structure induced on the boundary should also respect the properties of the corresponding conformal quasicrystal in a statistical sense, but we leave the investigation of this topic to future work.

[32] J. M. Luck, A Classification of Critical Phenomena on Quasicrystals and Other Aperiodic Structures, Europhys. Lett. 24, 359 (1993).

[33] M. Senechal, Quasicrystals and Geometry (Cambridge University Press, Cambridge, England, 1995).

[34] C. Janot, Quasicrystals: A Primer (Clarendon Press, Oxford, 1994).

[35] M. Baake and U. Grimm, Aperiodic Order: Volume 1: A Mathematical Invitation (Cambridge University Press, Cambridge, England, 2013).

[36] L. Boyle and P. J. Steinhardt, Self-Similar One-Dimensional Quasilattices, arXiv:1608.08220.

[37] L. Boyle and P. J. Steinhardt, Coxeter Pairs, Ammann Patterns and Penrose-like Tilings, arXiv:1608.08215.

[38] M. Gardner, Extraordinary Nonperiodic Tiling that Enriches the Theory of Tiles, Scientific American 236, 110 (1977).

[39] Strictly speaking, this is a $\tau$ quasicrystal of degree two since $\tau$ acts on two interval types; if $\tau$ acts on $n$ interval types, we would have a $\tau$ quasicrystal of degree $n$.

[40] E. Bombieri and J. E. Taylor, Which Distributions of Matter Diffract? An Initial Investigation, J. Phys. (Paris), Colloq. 47, C3 (1986); Quasicrystals, Tilings, and Algebraic Number Theory: Some Preliminary Connections, Contemp. Math. 64, 241 (1987).

[41] H. S. M. Coxeter, Regular Polytopes (Methuen, London, 1948). 
[42] R. Rietman, B. Nienhuis, and J. Oitmaa, The Ising Model on Hyperlattices, J. Phys. A 25, 6577 (1992).

[43] P. H. Ginsparg, Applied Conformal Field Theory, in Fields, Strings and Critical Phenomena (Les Houches, Session XLIX, 1988), edited by E. Brezin and J. Zinn-Justin (NorthHolland Publishing Company, Amsterdam, 1988).

[44] P. Di Francesco, P. Mathieu, and D. Senechal, Conformal Field Theory (Springer-Verlag, New York, 1997).

[45] W. Magnus, Noneuclidean Tesselations and Their Groups (Academic Press, New York and London, 1974).

[46] Note that, besides AdS/CFT, examples of holography include the Banks-Fischler-Shenker-Susskind matrix model [47] and the Klebanov-Strassler construction [48]. The ideas described in this paper relate to the AdS/CFT context, but we hope that progress in this context may lead to progress in the other holographic settings as well.
[47] T. Banks, W. Fischler, S. H. Shenker, and L. Susskind, $M$ Theory as a Matrix Model: A Conjecture, Phys. Rev. D 55, 5112 (1997).

[48] I. R. Klebanov and M. J. Strassler, Supergravity and a Confining Gauge Theory: Duality Cascades and $\chi S B$ Resolution of Naked Singularities, J. High Energy Phys. 08 (2000) 052.

[49] D. S. Rokhsar, D. C. Wright, and N. D. Mermin, The TwoDimensional Quasicrystallographic Space Groups with Rotational Symmetries Less Than 23-fold, Acta Crystallogr. Sect. A 44, 197 (1988).

[50] L. Nemeth, The Growing Ratios of Hyperbolic Regular Mosaics with Bounded Cells, Armenian J. Math. 9, 1 (2017).

[51] J. Weeks, KaleidoTile, a computer program for creating spherical, Euclidean, and hyperbolic tilings. 